Document downloaded from:

http://hdl.handle.net/10251/120653

This paper must be cited as:

Andres, B.; Ferrada, F.; Poler, R.; Camarinha-Matos, L. (2018). A Modeling Framework to Assess Strategies Alignment based on Collaborative Network Emotions. IFIP Advances in Information and Communication Technology. 534:349-361. https://doi.org/10.1007/978-3319-99127-6_30

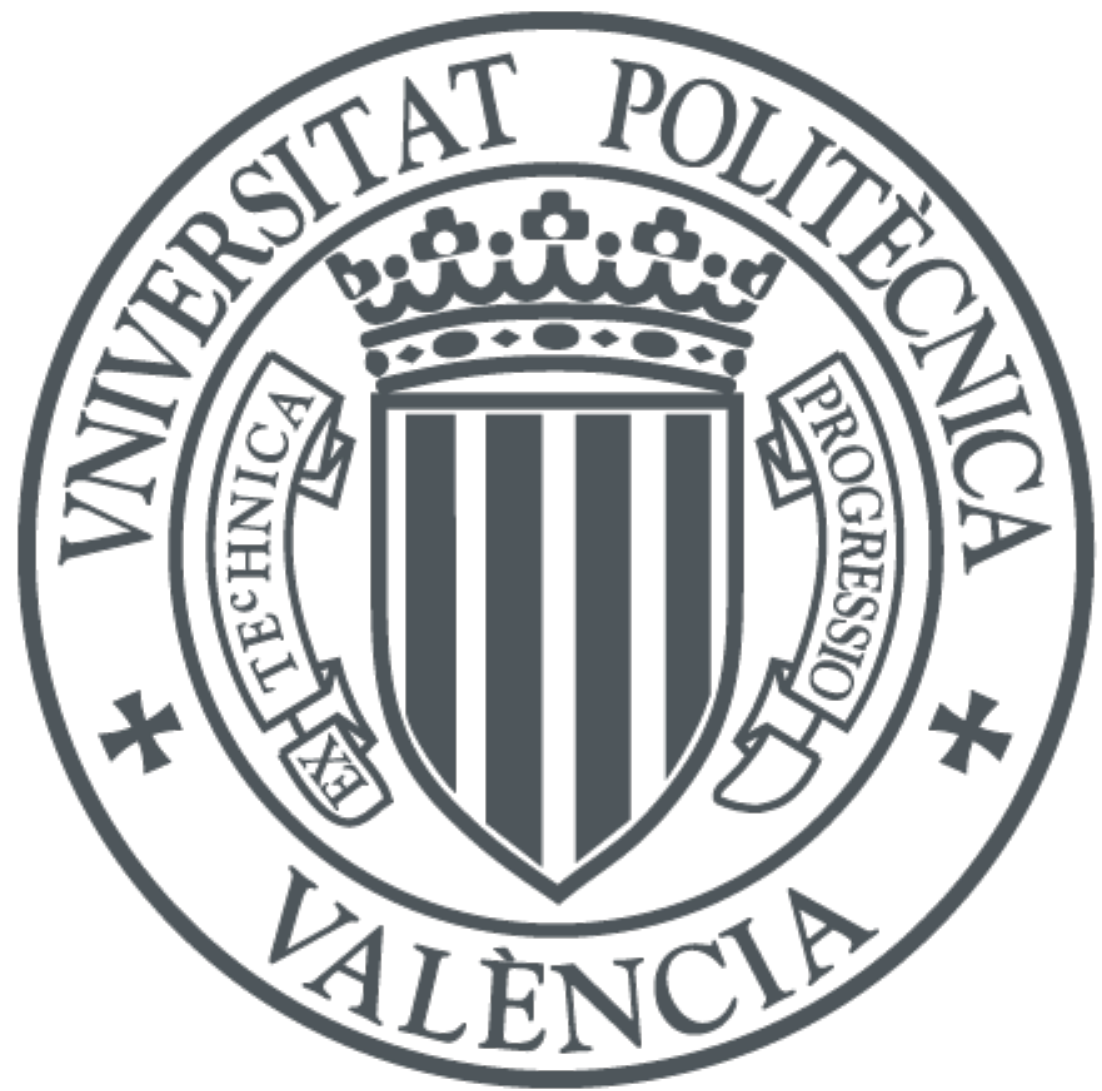

The final publication is available at

https://doi.org/10.1007/978-3-319-99127-6_30

Copyright Springer

Additional Information 


\title{
A Modeling Framework to Assess Strategies Alignment based on Collaborative Network Emotions
}

\author{
Beatriz Andres ${ }^{1}$, Filipa Ferrada ${ }^{2,3}$, Raul Poler ${ }^{1}$, Luis M. Camarinha-Matos ${ }^{2,3}$ \\ ${ }^{1}$ Research Centre on Production Management and Engineering (CIGIP), Universitat \\ Politècnica de València (UPV). Calle Alarcón, 03801 Alcoy, Spain \\ ${ }^{2}$ Faculty of Sciences and Technology, New University of Lisbon, Portugalis:pi \\ ${ }^{3}$ Uninova Institute, Centre of Technology and Systems, Portugal \\ $\{\underline{\text { bandres, }}, \underline{\text { rpoler}}\} @$ cigip.upv.es, $\{\underline{\mathrm{faf}}, \underline{\mathrm{cam}}\} @$ uninova.pt
}

\begin{abstract}
The Collaborative Networks (CN) discipline has been largely studied in last decades, addressing different problems and proposing solutions for the robust establishment of collaborative processes, within the enterprises willing to collaborate. The main aim of $\mathrm{CN}$ research is, therefore, to generate approaches that enable creating effective relationships in the long term, to achieve stable and agile alliances. The concept of alignment among the $\mathrm{CN}$ partners has been considered since the beginning of $\mathrm{CN}$ research. Nevertheless, novel perspectives of study in $\mathrm{CN}$, such as the consideration of collaborative emotional states, within the $\mathrm{CN}$, have been introduced in recent years. This paper connects the research area of strategies alignment and the $\mathrm{CN}$ emotion models. Accordingly, a modelling framework to assess strategies alignment considering the emotional environment within the $\mathrm{CN}$ is proposed. The modelling framework allows representing how the enterprises emotions affect in the selection and alignment of formulated enterprises' strategies.
\end{abstract}

Keywords: collaborative networks, strategies alignment, collaborative network emotions, system dynamics.

\section{Introduction}

Research works on the Collaborative Networks (CN) discipline has been increasing since its appearance, proof of this is the wide variety of articles published in the area [1]. As a result, a wide range of knowledge is available in the context of CN. This knowledge has been summarized in [2], through a taxonomic approach that gathers relevant collaborative processes, as well as models, guidelines and tools designed to support their proper execution. The proposed approaches, contributing to the research area of $\mathrm{CN}$, encompass collaborative processes from strategic to tactical and operational decision-making. The strategic decision-making level includes the following collaborative processes: coordination mechanisms design, decision system design, network design, partners' selection, partners' coordination and integration, performance management system design, product design, and strategy alignment. At the tactical decision-making level, the proposed approaches deal with contracts' negotiation, coordination mechanisms management, demand forecast, knowledge management, operations planning, performance management and measurement, costs 
and profits sharing, uncertainty management, etc. Finally, the collaborative processes at the operational decision-making level refer to information exchange management, interoperability, inventory management, lot sizing, order promising process, process connection, and scheduling.

The notion of alignment has been present since the origins of the $\mathrm{CN}$ discipline. This term is related with the integration and complementation of resources and capabilities of the different enterprises participating in the $\mathrm{CN}$ [3]. The main aim of the alignment is to achieve long term and sustainable collaborative relationships. In this regard, some authors have associated the idea of alignment with gaining better performance levels [4]. Accordingly, the alignment of CN partners has been studied in different areas, including value systems alignment [5] and strategies alignment [6][7].

Moreover, recent research in the discipline of $\mathrm{CN}$ is moving towards the need to provide socio-technical systems capable of recognizing the social and organizational complexity of the CN environment. In this direction, "human-tech" friendly systems resorting to cognitive models of human factors such as trust or emotion are being introduced. For instance, the collaborative emotion (C-EMO) modelling framework proposed in [8], adopts and integrates some models from the human psychology, sociology and affective computing areas. It consists on applying the emotional processes of humans to the $\mathrm{CN}$ context, creating the notion of "emotion" in $\mathrm{CN}$ and providing mechanisms to estimate the CN's and the organization's "emotional states" giving, in this way, support to decision-making processes.

In this context, this work is guided by the following research question "What would be a suitable framework to adequately support companies on modelling, assessment and resolution of the strategies alignment process from a collaborative perspective, considering the companies' collaborative emotional context?". In this line, the work presented in this paper, proposes a modelling framework for the assessment of strategies alignment having into account the emotional state of the collaborative environment and a simulation methodology based on system dynamics to model the inherent collaboration complexity. The proposed approach connects the research areas of collaborative enterprises' strategies alignment and the emotion models.

The remainder of this paper is organized as follows: Section 2 introduces the system dynamics simulation method, well regarded to model complex systems. Section 3 presents an overview and a relational view of the strategies alignment and the C-EMO models. Section 4 presents a modelling framework to assess the collaborative enterprises' strategies alignment regarding the emotional state. Section 5 includes a numerical example to provide an intuitive insight of the developed modelling framework and the derived simulation results. The work is concluded in Section 6, where future research lines are also identified.

\section{System Dynamics Simulation Methodology in CN}

The reason why analytical models are sometimes difficult to apply in complex systems, and particularly to $\mathrm{CN}$, is because mathematical formulae can be very complicated and interfere with finding a solution in a reasonable time [9]. In this 
regard, simulation methods are more fruitful when dealing with $\mathrm{CN}$, considering their capability of modelling suitable degrees of realism of the $\mathrm{CN}$ and attaining accurate system's description. CN are characterized by being complex systems formed by a diversity of autonomous and heterogeneous organizations, which are geographically distributed, and that collaborate to better achieve common or compatible goals [10]. This definition is analogue to the characterization of complex systems in system dynamics (SD) simulation methods: (i) decentralized nature, in which the system's behaviour arises from the self-organization of its components without these being controlled by any extrinsic entity to the system, (ii) the presence of loops of causality and nonlinear feedback, and (iii) the fact that it contains several self-contained units that can interact, evolve and adapt their behaviour to changes in the environment [11].

Moreover, with SD simulation, researchers can model and simulate interactions among different sub-systems leading in this way, to the overall system behaviour [12]. SD results in a different approach of the traditional if-then-else simulation methodologies; as such, it has been applied in many research fields, such as supply chain management and performance [13] [14], healthcare economic evaluation forecasting [15], biomedical sciences [16], partners selection [17], climate monitoring and water resource management [18], predicting social trends like technology adoption [19], and market changes forecasting [20].

Typically, SD simulation methods have been also applied jointly with multi-agent systems (MAS), with the aim of developing models in which a group of agents, individually and explicitly represented, interact in an environment in which certain variables evolve following a dynamic pattern [21].

\section{Background}

This section provides a brief overview of the strategies alignment model (SAM) and the C-EMO modelling framework. The adopted modelling approaches are given in both cases. An integrated view of both presented models is given, aiming at highlighting the associated theoretical body of knowledge with the intention of proposing a modelling framework to assess the strategies alignment considering $\mathrm{CN}$ emotions, as later described on section 4.

\subsection{Strategies Alignment Model}

Let us consider that two enterprises, a distributor $\left(e_{1}\right)$ and a manufacturer $\left(e_{2}\right)$ in a $\mathrm{CN}$, each one defines two objectives $\left(o_{i x}\right)$ and formulates two strategies $\left(S_{\mathrm{is}}\right)$. Each objective has an associated KPI to measure its performance. For instance, $e_{1}$ defines $o_{11}$ : Increase product sales by $10 \%$, and $o_{12}$ : Reduce product costs by $30 \%$; and formulates $S_{11}$ : Invest 0.5 m.u on marketing activities, and $S_{12}$ : Carry out negotiations with other manufacturers to reduce purchase costs. Similarly, $e_{2}$ defines $o_{21}$ : Increase the profit by $15 \%$, and $o_{22}$ : Reduce the amount of product that cannot be sold by $100 \%$; and formulates $S_{21}$ : Use different distribution channels to sell the product in 
other markets, and $S_{22}$ : Buy a machine to manufacture derivative products, reprocessing the product that cannot be sold (that is, low cost product). With this example it can be seen that $S_{12}$ is not compatible with $S_{21}$, because $S_{12}$ is dedicated to establishing new relationships with other manufacturers, which will imply reduction of the benefit defined in $o_{21}$. In addition, if $e_{1}$ conducts negotiations with other manufacturers $\left(S_{12}\right)$, the $o_{22}$ will be negatively influenced. Moreover, the activation of $S_{21}$ has a negative influence on $o_{11}$, defined to increase sales of the product; and the $o_{12}$ defined to reduce the costs of the product. Considering the above, $S_{12}$ and $S_{21}$ are identified as non-aligned strategies, if they are activated at the same time. On the other hand, $S_{11}$ and $S_{22}$ are considered as aligned strategies since they have a positive influence on the achievement of the defined objectives.

Carrying out collaboratively the strategies alignment process allows considering all objectives of the companies in the $\mathrm{CN}$ when deciding which strategies are the best to activate, achieving greater levels of adaptability, agility and competitiveness.

The SAM model [6] allows to formally represent the influences that the strategies activated in a company have on the performance indicators (KPI) defined to measure the achievement of the objectives, in all the $\mathrm{CN}$ companies. It will allow to model (i) the intra-enterprise influences, that is, the influences that the strategies formulated in a company have on the objectives defined in the same company; and (ii) interenterprise influences, that is, the influences that the strategies formulated in a company have on the objectives defined in the other companies of the $\mathrm{CN}$.

SAM's main objective is to identify, amongst all defined strategies, those that have a higher level of alignment. SAM calculates the improvement or worsening of the KPIs when a strategy is activated. Thus, the developed model supports companies in making decisions regarding the number of strategy units $\left(u \_S_{i s}\right)$ to be activated, and the time when these strategies must be activated $\left(t i S_{i s}\right)$ in order to maximize network's performance, $\left(K P I_{n e t}\right)$. Let's suppose that the strategy defined is, $S_{i s}$ : Buy machines (at maximum 3); translating this strategy to one unit of strategy, $u_{-} S_{i s}$ : Buy 1 machine; the activation of one unit of strategy $\left(u \_S_{i s}\right)$ has the associated cost of buying one machine (i.e. $S_{i s \_} m u=10000$ m.u.).

In order to model the process of strategies alignment, a set of five objects have been defined: (i) set of networks, net $=(1, \ldots, N E T)$; (ii) set of enterprises $i$ forming the network, $i=(1, \ldots, I)$; (iii) set of objectives $x$ defined by each enterprise, $x=(1, \ldots$, $X)$; (iv) set of key performance indicators (KPIs) $k$ to measure the level of objectives' achievement, $k=(1, \ldots, K)$; and (v) the set strategies $s$ formulated by each enterprise in order to achieve the objectives, $s=(1, \ldots, S)$. The influence that one strategy formulated by an enterprise $i\left(S_{i s}\right)$ has on a particular KPI defined by an enterprise $i$ to measure the objective $x_{i}\left(\mathrm{KPI}_{i x k}\right)$ is modelled through the function $f$ inf $S_{i s} K P I_{i x k}$ (see Fig. 1). This function, is a piecewise function that depends on the time $\left[f_{l}(t)\right]$. For modelling $f_{-}$inf_ $S_{i s_{-}} K P I_{i x k}$ a normalised horizon is used as the unit $(H=1)$ and the duration parameters are distributed in this $H$ of time: (i) $d_{1} S_{i s}$, delay, time period between the initial time of activation of $S_{i s}\left(t i S_{i s}\right)$ and the time when the $K P I_{i x k}$ is started to be influenced by the activated $S_{i s}$; (ii) $d_{2} S_{i s}$, time period between when $S_{i s}$ starts to influence the $K P I_{i x k}$ until the maximum level of influence is achieved (inf $S_{i s} K P I_{i x k}$ ); (iii) $d_{3} S_{i s}$, time period in which $S_{i s}$ is exerting the highest influence (inf $S_{i s}{ }_{-} P P I_{i x k}$ ) on the $K P I_{i x k}$; (iv) $d_{4} S_{i s}$, total duration of $S_{i s}$; (v) $t i S_{i s}$ identifies the starting point of activation of the $S_{i s}$ and allows modelling that not all the strategies 
are activated at the same time. Accordingly, the SAM allows identifying and assessing the strategies that are aligned and positively influence the objectives defined by all the network enterprises; enabling the increase of the network KPI.

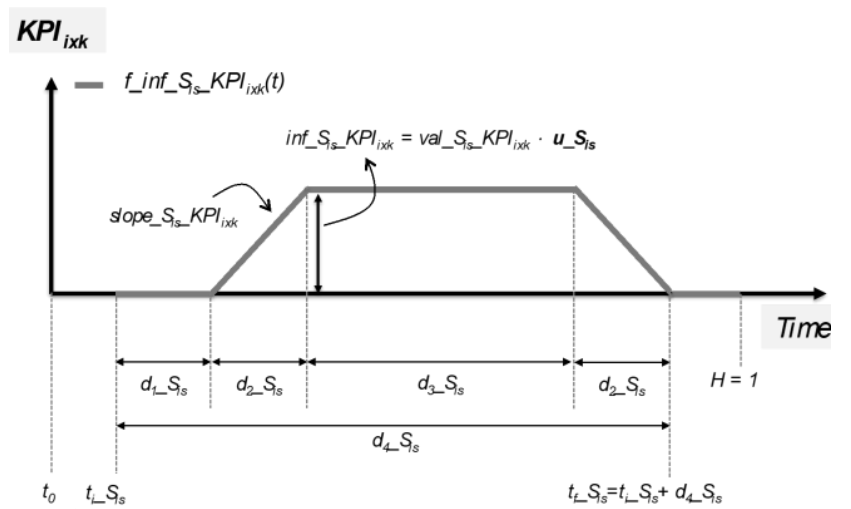

Fig. 1 Curve $\left(f_{-}\right.$inf $\left.S_{i s_{-}} K P I_{i x k}\right)$ that models the influence of $S_{i s}$ on the KPI $I_{i x k}$

\subsection{C-EMO Modeling Framework}

The Collaborative Emotion modeling framework (C-EMO), [22] represents a system that appraises emotions of $\mathrm{CN}$ members with different skills and characteristics and reasons about the way emotions affect those members and the entire collaborative environment. It gives support to the notion of emotions in the context of a $\mathrm{CN}$ and is grounded on the theories of human-emotion found in psychology and sociology. CEMO comprises the concepts of CNE (collaborative network emotion), IME (individual member emotion) and ANE (aggregated network emotion) and assumes the modeling of any typology of CN. C-EMO is built using object-oriented models providing two main constructs (IME and ANE models), each comprising its attributes and their relationships, as presented in Fig. 2: (i) Individual Member Emotion (IME model) for appraising the emotion of each $\mathrm{CN}$ member individually and examining the effects this emotion has on both the $\mathrm{CN}$ member behavior and the $\mathrm{CN}$ environment; and (ii) Aggregated Network Emotion (ANE model) for estimating the overall emotion present in the $\mathrm{CN}$ and examine the effects such emotion has on the network environment and on its members.

The theories that give support to the C-EMO are adapted from the theories of human emotion and consist of a combination of the dimensional theory and the components of emotion from the appraisal theory. The former is based on the Russel's circumplex model of emotion [15] which facilitated a good adaptation from the human model to the organizational model, through its well-defined structure for representing emotions. It comprises two dimensions of CNE: The Valence which is the dimension that represents the pleasure-displeasure continuum, and the Arousal that represents the level of activation, novelty and expectation of the emotional stimuli. Hence, CNEs can be defined with positive or negative valence and high or 
low arousal, and the emotions that are proposed to describe the "emotional states" of $\mathrm{CN}$ members are excitement (val $>0$, aro $>0)$, contentment $($ val $>0$, aro $<0)$, frustration $($ val $<0$, aro $>0)$ and depression $(v a l<0$, aro $<0)$. The four components of CNE which are based on the Scherer's [23] components of emotion, are: cognitive or appraisal component, feeling component, motivational component and expression component. Both the IME model and the ANE model building blocks represent the four CNE components.

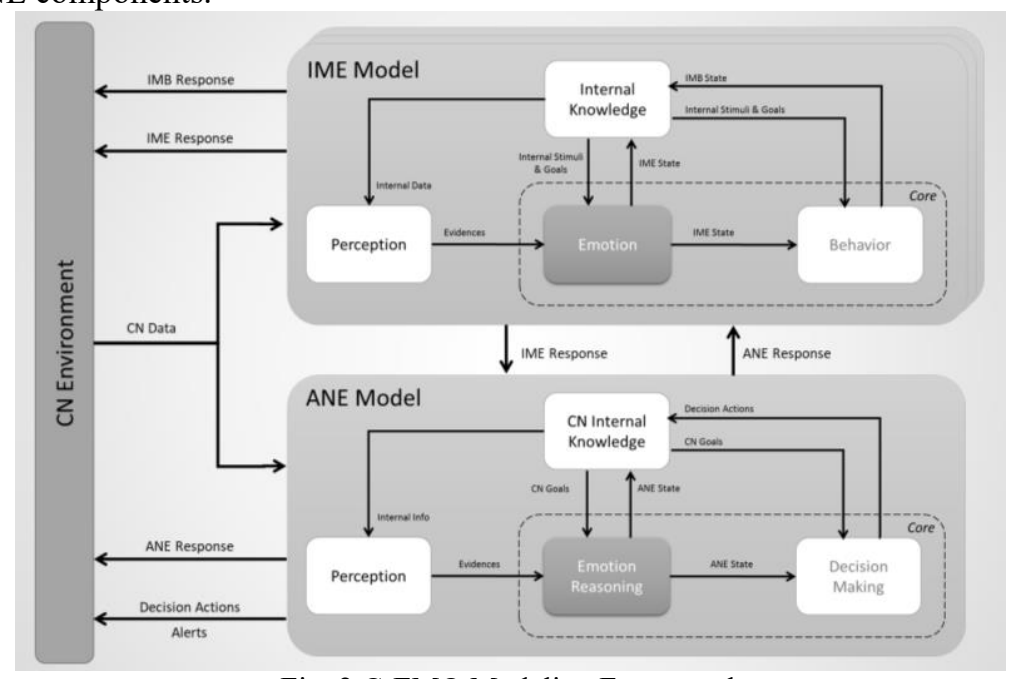

Fig. 2 C-EMO Modeling Framework

\subsection{Integrated View of SAM and C-EMO}

The diagram of Fig. 3 represents in a schematic way, an integrated view of the two approaches used in this paper, SAM and the C-EMO. Both approaches are linked through the degree of impatience $\left(\alpha_{i}^{S i s}\right)$, which is the output data of the C-EMO and is used as an input data of the SAM. Parameter $\alpha_{i}^{\text {Sis }}$ is widely explained in section 4, but roughly speaking, the degree of impatience is the extent of rapidness into which the enterprise is willing to obtain the maximum level of $K P I_{i x k}$. The $\alpha_{i}^{S i s}$ is generated in the IME model of the C-EMO, which is modelled in the Emotion element using the SD method. Values of $\alpha_{i}^{\text {Sis }}$ near 0 indicate that the enterprise $i$ is more patient to obtain the expected level of $K P I_{i x k}$, when the strategy $S_{i s}$ is activated. Parameter $\alpha_{i}^{S i s}$ is an input to the SAM and is used through translating $\alpha_{i}^{S i s}$ in time units $\left(t_{-} S_{i s}\right)$, when the $S_{i s}$ is activated. Moreover, SAM calculates the $t_{m_{-}} S_{i s}$, which is the actual time when the maximum level of the KPI ixk is achieved, being $S_{i s}$ activated. In order to consider the impatience emotion in SAM, the proposed extension of SAM considers the comparison between the $t_{-} S_{i s}$ (obtained from $\alpha_{i}^{S i s}$ ) and $t_{m} S_{i s}$. Accordingly, if the time in which the enterprise $i$ is willing to achieve the maximum level of $K P I_{i x k}$ is higher than the real time in which the maximum level of the $K P I_{i x k}$ is achieved $\left(t S_{i s}>t_{m} S_{i s}\right)$, then the strategy $S_{i s}$ is activated; and therefore, aligned with all the KPIS defined by each of the $\mathrm{CN}$ partners, and with the impatience emotion of all the $\mathrm{CN}$ partners. Otherwise, when $t_{-} S_{i s}<t_{m_{-}} S_{i s}$, the $S_{i s}$ will not be activated. Finally, the extended 
SAM, will allow to identify the amount of extra monetary units that the enterprise $i$ can invest in each strategy $\mathrm{Sis}$, in order obtain in a quicker way the maximum level of $K P I_{i x k}$, and comply with the condition $t_{-} S_{i s}>t_{m} S_{i s}$. It is assumed that, the higher amount of extra monetary units invested implies a lower time needed to obtain the maximum level of $K P I_{i x k}$. Thus, if the enterprise is very impatience (higher values of $\alpha_{i}^{S i s}$ ), this is translated in a lower $t \_S_{i s}$, therefore more extra monetary units will be need to invest.

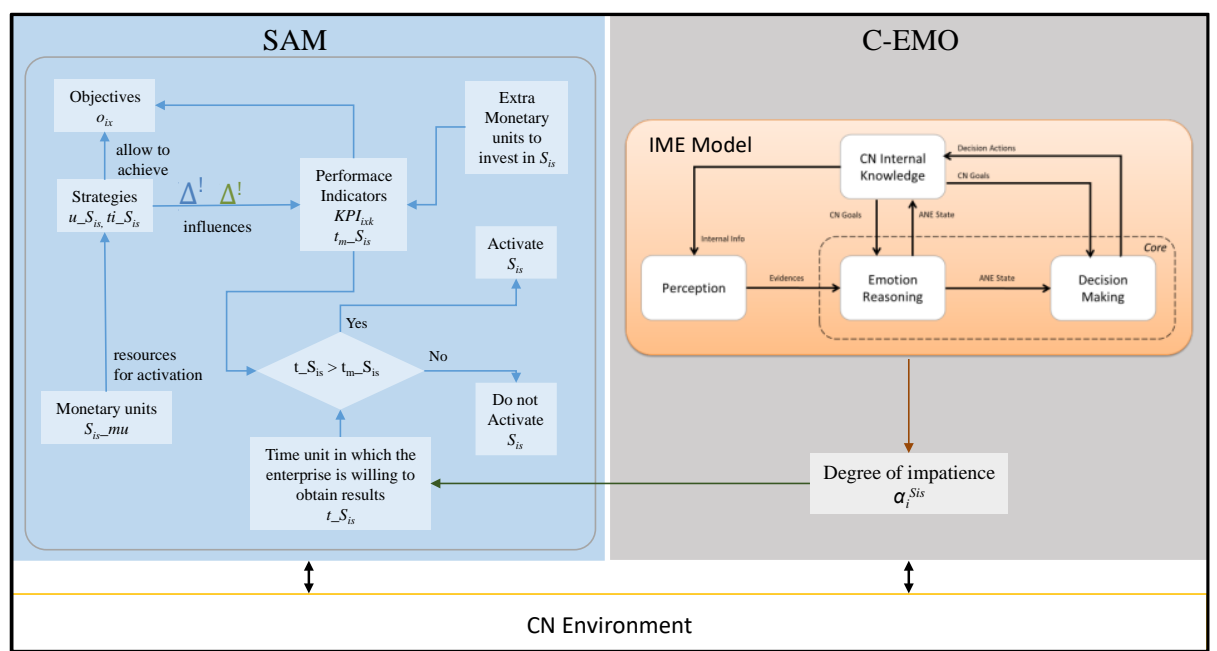

Fig. 3. Integrated view of SAM and the IME model of the C-EMO modelling framework

\section{Modelling Framework}

The proposed modelling framework uses as a base the SAM model, extending it for the consideration of emotions when a strategy is collaboratively activated between partners of a CN. The C-EMO modelling framework, as previous described, considers gathering all the affective states from two variables Valence and Arousal. Accordingly, the IME can be understood as a linear combination of these two dimensions and the emotional state of an enterprise is described as ei(t) = $<V i(t), A i(t)>$. The IME, therefore depends on the time. Following the CNE dimensional approach, there is one possible active emotion for each circumplex quadrant: excitement, contentment, frustration and depression. In order to connect both models, the IME model of C-EMO and SAM, a new combination of valence and arousal is introduced in order to define the patience and impatience emotions. In the same way, the IME model, via its system dynamics model, is adjusted to comprise the new modelling parameters and appraise the new emotions. The emotion impatience, is then used as an input to the extended SAM. The degree of impatience is related with the time unit, in which each $\mathrm{CN}$ partner is willing to achieve the maximum level of $K P I_{i x k}$, when a particular strategy $S_{i s}$ is activated. 
First, it must be stated that strategies can be defined at short, medium or long term: (i) short term (ST): actions that are designed with the aim of obtaining immediate results, between one day and, at the most, one month; e.g. an advertisement campaign in the media; (ii) medium term (MT): actions to remain active without despairing in shortterm action or falling into the passivity of the long term; e.g. collaborative distribution, through contacting related distribution companies and reaching an agreement to cross-ship products to open a wider distribution channel and attract potential customers in the short term; and (iii) long term (LT): actions designed and conceptualized with a vision for the future, whose positive results do not begin to be noticed until after 6 months and a year since their implementation, but they bring a lot of value and last in the future; e.g. an $R+D$ action to create a new product.

Second, the degree of impatience is estimated through the proposed extension of the IME model. The result of IME consists of a value of $\alpha_{i}^{\text {Sis }}$ that varies from 0 to 1 , so that $\alpha_{i}^{S i s}=[0,1]$; being $\alpha_{i}^{\text {Sis }}$ a real number. The $\alpha_{i}^{\text {Sis }}$ is related with the time that the enterprise $e_{i}$ is willing to wait, when the strategy is activated, until it obtains the maximum level of $K P I_{i x k}$. Parameter $\alpha_{i}^{S i s}$ is directly related with the horizon defined in the SAM, which is normalised, $H=1$, and is inverse to $t_{-} S_{i s}$; thus, $t_{-} S_{i s}=1-\alpha_{i}^{\text {Sis }}$ :

- $\alpha_{i}^{S i s}=0\left(t \_S_{i s}=1\right)$ indicates that the enterprise $e_{i}$ does have a lot of patience, so that once the strategy is activated, the enterprise is not worried about the time in which it will obtain the maximum level of $K P I_{i x k}$. This may cause a problem because once the strategy $S_{i s}$ is activated, and the enterprise is investing on $S_{i s}$, it could happen that $S_{i s}$ does not arrive to generate a good level of performance $\left(K P I_{i x k}\right)$. This could imply that the enterprise is losing money while waiting for $S_{i s}$ to start generating results (increase the $K P I_{i x k}$ ).

- $\alpha_{i}^{S i s}=1\left(t S_{i s}=0\right)$ indicates that $e_{i}$ does not have patience, so that once the strategy is activated, the enterprise is willing to obtain the desired results immediately. It is characterised by the fact that the enterprise does not see the results, of the activated strategy, immediately, and stops the investment. This may cause a problem because it could happen that the results (achieve the higher level of $\left.K P I_{i x k}\right)$ are going to be visible in a near time, but not immediately. The enterprise can lose the investment, but if it would wait a little bit, the results could start to be visible, and the maximum level of the $K P I_{i x k}$ could be achieved.

- $0<\alpha_{i}^{S i s} \leq 0,5\left(1>t_{-} S_{i s} \geq 0,5\right)$ means that $e_{i}$ is not as passive as when $\alpha_{i}^{S i s}=0$.

- $0,5<\alpha_{i}^{S i s}<1\left(0, \overline{5}>t S_{i s}>0\right)$ means that $e_{i}$ is not as restlessness and impetuousness as when $\alpha_{i}^{S \overline{s i s}}=1$.

Finally, the degree of impatience of each enterprise $\mathrm{e}_{i}\left(\alpha_{i}^{S i s}\right)$ is also defined according to the three types of strategies (ST, MT and LT). C-EMO models the degree of impatience in a specific enterprise depending on the type of strategies defined at the short, medium and long term. It could happen that the enterprises are less patience when formulating short term strategies, because they manifest quickly their results; and the enterprises are more patience when formulating long term strategies, because the results need more time to be reflected in the KPIs:

- $\alpha_{i}^{S i s} S T$ : degree of impatience when $e_{i}$ formulates $S_{i s}$ in the short term;

- $\alpha_{i}^{S i s}{ }_{-}^{M T}$ : degree of impatience when $e_{i}$ formulates $S_{i s}$ in the medium term;

- $\alpha_{i}^{S i s \_L T}$ : degree of impatience when $e_{i}$ formulates $S_{i s}$ in the long term.

In order to answer the research question presented in Section 1, the SAM is extended by considering the following flow diagram (Fig. 4); the shadowed squares 
mark the proposed extensions. The notation and formulas (Table 1), which relate the emotion perspective with the SAM are also described.

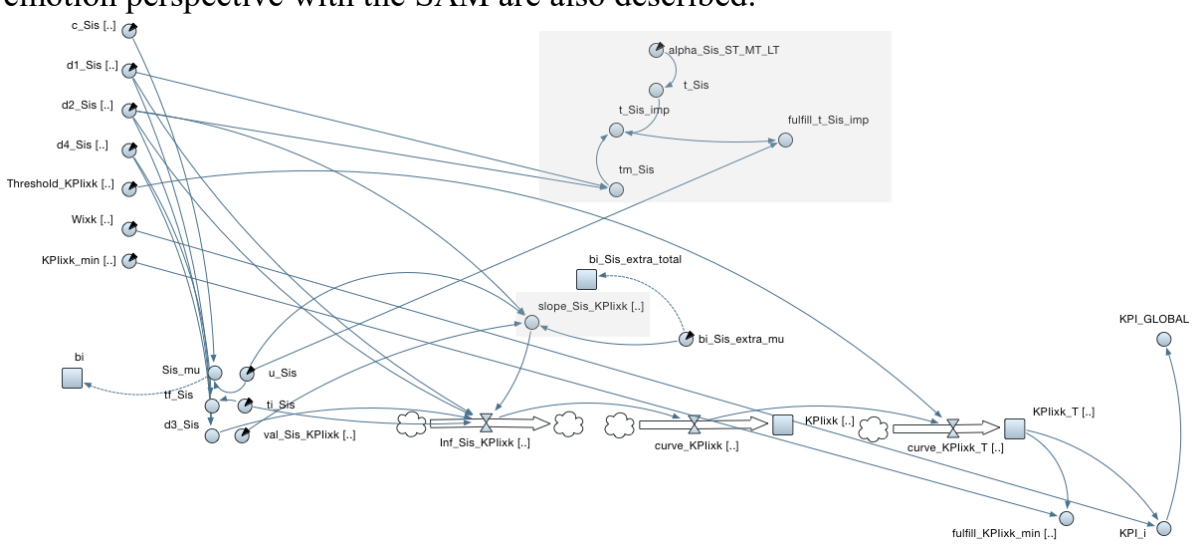

Fig. 4. Extended SAM: Flow diagram

Table 1. Nomenclature and SD formulation

\begin{tabular}{|c|c|}
\hline \multicolumn{2}{|l|}{ Nomenclature } \\
\hline Parameter & Definition \\
\hline bi_Sis_extra_mu & $\begin{array}{l}\text { number of extra monetary units that the enterprise } i \text { invest in each strategy } S_{i s} \\
\text { formulated }\end{array}$ \\
\hline alpha_Sis_ST & $\begin{array}{l}\text { degree of impatience when the strategy } S_{\text {is }} \text { is characterized by being for the short } \\
\text { term }[0,1] \text {. }\end{array}$ \\
\hline alpha_Sis_MT & $\begin{array}{l}\text { degree of impatience when the strategy } S_{i s} \text { is characterized by being for the } \\
\text { medium term }[0,1]\end{array}$ \\
\hline alpha_Sis_LT & $\begin{array}{l}\text { degree of impatience when the strategy } S_{i s} \text { is characterized by being for the long } \\
\text { term }[0,1]\end{array}$ \\
\hline u_Sis & units of strategy $S_{i s}$ activated \\
\hline Dynamic variable & Definition \\
\hline slope_Sis_KPIixk & $\begin{array}{l}\text { slope of the ramp in represented in } f \text { inf } S_{i s} K P I_{i k k}(t) \text { multiplied by the number of } \\
\text { extra monetary units that the enterprise } i \text { invest in each strategy } S_{i s} \text { formulated }\end{array}$ \\
\hline$t m \_S i s$ & $\begin{array}{l}\text { time in which the maximum level of the } K P I_{i x k} \text { is achieveed when the } S_{\text {is }} \text { is } \\
\text { activated (t.u.) }\end{array}$ \\
\hline t_Sis_imp & $\begin{array}{l}\text { time in which the enterprise is willing to achieve the maximum level of } K P I_{i x k} \text {, } \\
\text { when the } S_{\text {is }} \text { is activated (t.u.) }\end{array}$ \\
\hline$t$ _Sis & $\begin{array}{l}\text { time that relates the degree of impatience with the time in which the enterprise is } \\
\text { willing to achieve the maximum level of } K P I_{i x k} \text {, when the } S_{\text {is }} \text { is activated (t.u.) }\end{array}$ \\
\hline $\begin{array}{l}\text { fulfill_t_Sis_imp } \\
\text { Stock variable }\end{array}$ & $\begin{array}{l}1 \text { when }\left(t \text { SSis_imp }{ }^{*} u_{-} \text {Sis }\right)>=0 ; 0 \text { otherwise } \\
\text { Definition }\end{array}$ \\
\hline bi_Sis_extra_total & $\begin{array}{l}\text { extra monetary units that the enterprise } i \text { is willing to invest at maximum in each } \\
\text { strategy } S_{\text {is }} \text { formulated }\end{array}$ \\
\hline \multicolumn{2}{|l|}{ SDFormulation } \\
\hline \multicolumn{2}{|r|}{ 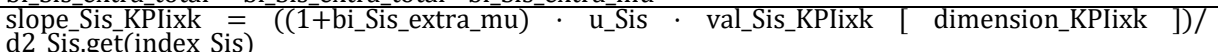 } \\
\hline $\begin{array}{l}\mathrm{tm} \text { t_Sis }=\mathrm{d} 1 \text { 1_Sis.get(index_Sis) }+ \text { d2_Sis.get(index_. } \\
\mathrm{t} \text { Sis imp }=\mathrm{t} \text { Sis-tm Sis }\end{array}$ & ex_Sis) + d2_Sis.get(index_. \\
\hline
\end{tabular}

The parameter bi_Sis_extra_mu acts as a new decision variable of the SAM, identifying the number of extra monetary units that enterprise $i$ has to invest in each formulated strategy $S_{\text {is, }}$, apart from the budget initially defined. The slope of function $f$ inf_S $S_{i s_{-}} K P I_{i x k}(t)$ is higher because it is multiplied by the extra budget used (bi_Sis_extra_mu); therefore, more quickly reaches the maximum level of $K P I_{i x k}$. But also, as the enterprise invests more money for the activation of the strategy $S_{i s}$, the maximum level of $K P I_{i x k}$ also increases. In the extension of SAM it is considered that 
the relation between the bi_Sis_extra_mu and the $K P I_{i x k}$ is $1: 1$. When the strategy $S_{i s}$ has a positive influence in the $K P I_{i x k}$, the more extra money invested in a particular strategy (bi_Sis_extra_mu), the higher level of $K P I_{i x k}$ is reached. If the influence is negative, the higher extra money invested, the lower level of $K P I_{i x k}$ is reached. The extended SAM also includes the restrictions related with the parameters bi_Sis_extra_total and t_Sis_imp, which must be positive, and the parameter fulfill_t_Sis_imp, which must be equal to 1 .

\section{Case Study}

An example is presented with the main aim of illustrating the proposed approach. This example considers two enterprises ( $e_{1}$ and $e_{2}$ ), each one defining two objectives $\left(e_{1}: o_{11}\right.$ and $o_{12} ; e_{2}: o_{12}$ and $o_{22}$ ): (i) $o_{11}$ : Increase the product sales by a $10 \%$; (ii) $o_{12}$ : Reduce the costs of the product by a $5 \%$; (iii) $o_{21}$ : Increase the enterprise profit by a 15\%; (iv) oz2: Reduce the quantity of product that cannot be sold by $100 \%$. The achievement of the objectives is measured through the $K P I_{i x k}$. In order to achieve the objectives, each enterprise formulates two strategies ( $e_{1}: S_{11}$ and $S_{12} ; e_{2}: S_{21}$ and $S_{22}$ ), with different levels of application: (i) $S_{11}$ : Increase the marketing activities on the product (ST); (ii) $S_{12}$ : Conduct negotiations with the manufacturing partner to reduce purchasing costs (LT); (iii) $S_{21}$ : Open new distribution channels to spread out the product in other markets (MT); (iv) $S_{22}$ : Product promotions (ST). The input data gathered directly from the enterprises is stored in Table 2 , in which the data related with the strategies durations and costs can be seen as well as the values of influence val_ $S_{i s_{-}} K P I_{i x k}$ estimated by each enterprise and the budget.

Table 2. Input data

\begin{tabular}{|c|c|c|c|c|c|c|c|c|c|c|c|}
\hline \multirow{2}{*}{\multicolumn{11}{|c|}{ Enterprise $1 \quad b_{1}=3$}} & \\
\hline & & & & & & & & $k p i_{111}$ & & $k p i_{121}$ & \\
\hline \multirow[t]{3}{*}{\begin{tabular}{|l|l}
$S_{11}$ \\
\end{tabular}} & $u_{-} S_{11}$ & \begin{tabular}{|l|l|}
$? i_{-} S_{11}$ \\
\end{tabular} & $?$ & $c_{-} S_{11}$ & 2 & \begin{tabular}{|l|l|}
$d_{1} s_{11}$ & 0,05 \\
\end{tabular} & \begin{tabular}{|l|l|l|l|}
$\boldsymbol{d}_{2}-\boldsymbol{S}_{11}$ & 0,01 & $\boldsymbol{d}_{4} \boldsymbol{S}_{\mathbf{2 1}}$ & 0,6 \\
\end{tabular} & $w_{111}$ & 0,5 & $w_{121}$ & 0,5 \\
\hline & b1__S $S_{1 \_ \text {extra_mu }}$ & \begin{tabular}{|l|l|} 
? & alpha_ $S_{t t_{-}} S T$ \\
\end{tabular} & 0,1 & \begin{tabular}{|l|}
$b 1_{-} S_{11}$ extra_total \\
\end{tabular} & 10 & & & \begin{tabular}{|l|} 
Threshold_kpi $i_{111}$ \\
\end{tabular} & 0,2 & Threshold_kpi $i_{121}$ & 0,1 \\
\hline & & & & & & & & val_s $S_{11-}$ kpi $i_{111}$ & 0,8 & $v a l S_{11} k p i_{121}$ & $-0,01$ \\
\hline \multirow[t]{5}{*}{\begin{tabular}{|l|l|l|l|l}
$S_{12}$ & \\
\end{tabular}} & $u_{-} S_{12}$ & \begin{tabular}{|l|l|}
$?$ & $t i_{1} S_{12}$ \\
\end{tabular} & $?$ & $c_{-} S_{12}$ & 3 & \begin{tabular}{|l|l|}
$d_{1} \boldsymbol{S}_{12}$ & 0,2 \\
\end{tabular} & \begin{tabular}{|l|l|l|l|}
$\boldsymbol{d}_{2} \boldsymbol{S}_{12}$ & 0,03 & $\boldsymbol{d}_{4} \boldsymbol{S}_{2}$ & 0,5 \\
\end{tabular} & val_S $S_{12} k p i_{111}$ & 0,3 & val__s $S_{12} k_{p i i_{121}}$ & 0,7 \\
\hline & b1__S I2_extra_mu $_{1}$ & \begin{tabular}{|l|l|}
$?$ & alpha $S_{22} M T$ \\
\end{tabular} & 0,9 & \begin{tabular}{|l|}
$b 1 S_{1} S_{12}$ extra_total \\
\end{tabular} & 10 & & & val__ $S_{21} k p i_{111}$ & $-0,1$ & val_s $S_{21} k p i_{121}$ & $-0,3$ \\
\hline & & & & & & & & val__s $S_{22} k p i_{111}$ & 0,3 & $v a l \_S_{22-} k p i_{121}$ & 0,2 \\
\hline & & & & & & Enterpri & ise $2 \quad b 2=6$ & & & & \\
\hline & & & & & & & & $k p i_{211}$ & & $k p i_{221}$ & \\
\hline \multirow[t]{3}{*}{\begin{tabular}{|l|}
$S_{21}$ \\
\end{tabular}} & $u_{-} S_{21}$ & \begin{tabular}{|l|l|}
$?$ & $t i_{-} S_{21}$ \\
\end{tabular} & $?$ & $c_{-} S_{21}$ & 6 & 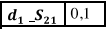 & \begin{tabular}{|l|l|l|l|l|}
$\boldsymbol{d}_{2} \boldsymbol{S}_{21}$ & 0,02 & $\boldsymbol{d}_{4} \boldsymbol{S}_{2 \mathbf{2 1}}$ & 0,75 \\
\end{tabular} & $w_{211}$ & 0,5 & $w_{221}$ & 0,5 \\
\hline & b2_-S si_extra_mu & \begin{tabular}{|l|l|}
$?$ & alpha_ $S_{12} L T T$ \\
\end{tabular} & 0,6 & b2 $2_{-} S_{12}$ extra_total & 10 & & & \begin{tabular}{|l} 
Threshold_kpi $i_{211}$ \\
\end{tabular} & 0,3 & Threshold_kpi $i_{221}$ & 0,15 \\
\hline & & & & & & & & val_S $S_{21}$ kpi $i_{211}$ & 0,7 & val_ $S_{21} k p i_{221}$ & 0 \\
\hline \multirow{3}{*}{\begin{tabular}{|l|}
$S_{22}$ \\
\end{tabular}} & $u_{-} S_{22}$ & \begin{tabular}{|l|l|}
$? i_{-} S_{22}$ \\
\end{tabular} & $?$ & $c_{-} S_{22}$ & \begin{tabular}{|l|l}
5 \\
\end{tabular} & \begin{tabular}{|l|l|}
$d_{1} S_{21}$ & 0,05 \\
\end{tabular} & \begin{tabular}{|l|l|l|l|}
$d_{2-} S_{21}$ & 0,01 & $d_{4}-S_{21}$ & 0,5 \\
\end{tabular} & val__S $S_{22}-k p i_{211}$ & 0,2 & val__ $S_{22-} k_{p i} i_{221}$ & 0,8 \\
\hline & b2_-S s2_extra_mu & \begin{tabular}{|l|l|} 
? & alpha_ $S_{22} S T$ \\
\end{tabular} & 0,95 & bi__S $S_{22}$ extra_total & 10 & & val_S $S_{11} k p i_{211}$ & 0,3 & val_S $S_{11}$ kppi $i_{221}$ & 0,4 \\
\hline & & & & & & & & val__ $s_{12} k$ kpi $i_{211}$ & $-0,2$ & val__ $S_{12}$ k $k p i_{221}$ & $-0,3$ \\
\hline
\end{tabular}

Two scenarios are compared for the validation of the SAM using C-EMO. Scenario 1 uses data of Table 2, and enterprise 2 identifies an alpha_S22_ST $=0,95$. In scenario 2 the enterprise 2 reduces by 0,05 the degree of impatience, so that alpha_S22_ST $=0,9$. Results (Table 3) with the initial input data (scenario 1) show that the $S_{11}$ and $S_{21}$ must be activated in one unit, providing a KPI of the network equal to 2,6315. The solution in scenario 2, shows that the $S_{11}$ and $S_{22}$ must be activated in one unit, giving a KPI of the network equal to 3,927; a $37 \%$ higher than in the scenario 1. Therefore the proposed approach, allows to support the $\mathrm{CN}$ enterprises, advising that slight differences in the degree of impatience could change 
the activation of aligned strategies increasing on higher values of enterprise and network KPIs.

Table 3. Results

\begin{tabular}{l|l|l|l|l|l|l|l|l|l|l|l|l|l|l}
\hline Scenario 1 & u_S $\mathrm{S}_{11}$ & 1 & $\mathrm{u} \_\mathrm{S}_{12}$ & 0 & $\mathrm{u} \_\mathrm{S}_{21}$ & 1 & $\mathrm{u} \_\mathrm{S}_{22}$ & 0 & $\mathrm{KPI}_{1}$ & 0,907 & $\mathrm{KPI}_{2}$ & 4,356 & $\mathrm{KPI}_{\text {net }}$ & 2,6315 \\
\hline Scenario 2 & $\mathrm{u} \_\mathrm{S}_{11}$ & 1 & $\mathrm{u} \_\mathrm{S}_{12}$ & 0 & $\mathrm{u} \_\mathrm{S}_{21}$ & 0 & $\mathrm{u} \_\mathrm{S}_{22}$ & 1 & $\mathrm{KPI}_{1}$ & 3,479 & $\mathrm{KPI}_{2}$ & 4,375 & $\mathrm{KPI}_{\text {net }}$ & 3,927 \\
\hline
\end{tabular}

\section{Conclusions}

A connection between two relevant approaches in the scope of $\mathrm{CN}$ discipline is presented, aiming at proposing a new modelling framework to support companies on modelling, assessment and resolution of the strategies alignment process considering their collaborative emotional state. Both approaches are based on SD simulation method. An integrated view diagram relating SAM and C-EMO models is proposed. It starts with the calculation of the degree of impatience within the IME model of CEMO. The degree of impatience is translated in time units and serves as an input to SAM, which identifies the strategies to be activated accordingly and computes the extra monetary units to be invested in the strategies execution in order to accelerate obtaining the maximum level of $K P I_{\text {ixk }}$ monetary.

Future work will rely on extending the proposed approach, from a decentralized perspective. Accordingly, future developments will pass by jointly using system dynamics and agent-based $(\mathrm{AB})$ simulation methodologies. With the introduction of $\mathrm{AB}$, the proposed approach will work at two levels, (i) at the $\mathrm{CN}$ level, modelling the complex system as a collection of autonomous decision making entities called agents, and; (ii) at the individual level, where each agent individually evaluates its situation and takes decisions following a set of rules that are modeled with the system dynamics method.

\section{References}

[1] L. M. Camarinha-Matos, "Collaborative Networks in Industry and the role of PRO-VE," Int. J. Prod. Manag. Eng., vol. 2, no. 2, pp. 53-57, 2014.

[2] B. Andres and R. Poler, "Models, guidelines and tools for the integration of collaborative processes in non-hierarchical manufacturing networks : a review," Int. J. Comput. Integr. Manuf., vol. 2, no. 29, pp. 166-201, 2016.

[3] U. Bititci, V. Martinez, P. Albores, and J. Parung, "Creating and Managing Value in Collaborative Networks," Int. J. Phys. Distrib. Logist. Manag., vol. 34, no. 3/4, pp. 251268, 2004

[4] B. Carbo, "Align the Organization for Improved Supply Chain Performance.," ASCET Proj., vol. 2, pp. 244-447, 2002.

[5] P. Macedo and L. Camarinha-Matos, "Value Systems Alignment Analysis in Collaborative Networked Organizations Management," Appl. Sci., vol. 7, no. 12, p. 123, 2017.

[6] B. Andres and R. Poler, "A decision support system for the collaborative selection of strategies in enterprise networks," Decis. Support Syst., vol. 91, pp. 113-123, 2016.

[7] B. Andres, P. Macedo, L. M. Camarinha-Matos, and R. Poler, "Achieving Coherence 
between Strategies and Value Systems in Collaborative Networks," IFIP Adv. Inf. Commun. Technol., vol. 434, pp. 261-272, 2014.

[8] F. Ferrada and L. M. Camarinha-Matos, "A System Dynamics and Agent-Based Approach to Model Emotions in Collaborative Networks," DoCEIS 2017, IFIP AICT, vol. 499, pp. 29-43, 2017.

[9] F. Campuzano and J. Mula, Supply Chain Simulation. A System Dynamics Approach for Improving Performance. Springer London Dordrecht Heidelberg New York, 2011

[10] L. M. Camarinha-Matos and H. Afsarmanesh, "Collaborative networks : a new scientific discipline," pp. 439-452, 2005.

[11] T. Vicsek, "Complexity: The bigger picture," Nature, vol. 418, no. 6894, p. 131, 2002.

[12] J. Sterman, G. Richardson, and P. Davidsen, "Modelling the Estimation of Petroleum Resources in the United States," Technol. Forecast. Soc. Chang., vol. 33, no. 3, pp. 219249, 1998.

[13] D. Vlachos, P. Georgiadis, and E. Iakovou, "A system dynamics model for dynamic capacity planning of remanufacturing in closed-loop supply chains," Comput. Oper. Res., vol. 34, no. 2, pp. 367-394, 2007.

[14] F. Campuzano-Bolarín, J. Mula, and D. Peidro, "An extension to fuzzy estimations and system dynamics for improving supply chains," Int. J. Prod. Res., vol. 51, no. 10, pp. 3156-3166, 2013.

[15] P. Barton, S. Bryan, and S. Robinson, "Modelling in the economic evaluation of health care: selecting the appropriate approach," J. Heal. Serv. Res. Policy, vol. 9, no. 2, pp. 110118,2004

[16] T. Eldabi, R. J. Paul, and T. Young, "Simulation Modelling in Healthcare: Reviewing Legacies and Investigating Futures," J. Oper. Res. Soc. Spec. Issue Oper. Res. Heal., vol. 58, no. 2, pp. 262-270, 2007.

[17] B. Andres, R. Poler, L. M. Camarinha-Matos, and H. Afsarmanesh, "A simulation approach to assess partners selected for a collaborative network," Int. J. Simul. Model., vol. 16, no. 3, 2017.

[18] A. Gohari, A. Mirchi, and K. Madan, "System Dynamics Evaluation of Climate Change Adaptation Strategies for Water Resources Management in Central Iran," Water Resour. Manag., vol. 31, no. 5, pp. 1413-1434, 2007.

[19] D. Fishera, J. Norvell, S. Sonka, and M. J. Nelson, "Understanding technology adoption through system dynamics modeling: implications for agribusiness management,"," Int. Food Agribus. Manag. Rev., vol. 3, pp. 281-296, 2000.

[20] J. M. Lyneisa, "System dynamics for market forecasting and structural analysis," Syst. Dyn. Rev., vol. 16, no. 1, pp. 3-25, 2000.

[21] A. Borshchev and A. Filippov, "From System Dynamics and Discrete Event to Practical Agent Based Modeling: Reasons, Techniques, Tools," in The 22nd International Conference of the System Dynamics Society, 2004.

[22] F. Ferrada, "C-EMO: A Modeling Framework for Collaborative Network Emotions Doctoral dissertation, Nova University of Lisbon, Portugal, 2017. Retrieved from: https://run.unl.pt/handle/10362/26857," 2017.

[23] K. R. Scherer, "Emotions are emergent processes: they require a dynamic computational architecture," Rev. of. Philos. Trans. R. Soc. Biol. Sci., vol. 364, no. 1535, pp. 3459-3474, 2009. 